This item was submitted to Loughborough's Research Repository by the author.

Items in Figshare are protected by copyright, with all rights reserved, unless otherwise indicated.

\title{
A single representation to support assembly and process planning in feature- based design machined parts
}

PLEASE CITE THE PUBLISHED VERSION

http://dx.doi.org/10.1243/0954405991517326

PUBLISHER

Sage / @ IMechE

VERSION

AM (Accepted Manuscript)

LICENCE

CC BY-NC-ND 4.0

\section{REPOSITORY RECORD}

Case, Keith, and Wan Abdul Rahman Jauhari Bin Wan Harun. 2019. "A Single Representation to Support Assembly and Process Planning in Feature-based Design Machined Parts”. figshare.

https://hdl.handle.net/2134/13957. 
This item was submitted to Loughborough's Institutional Repository (https://dspace.lboro.ac.uk/) by the author and is made available under the following Creative Commons Licence conditions.

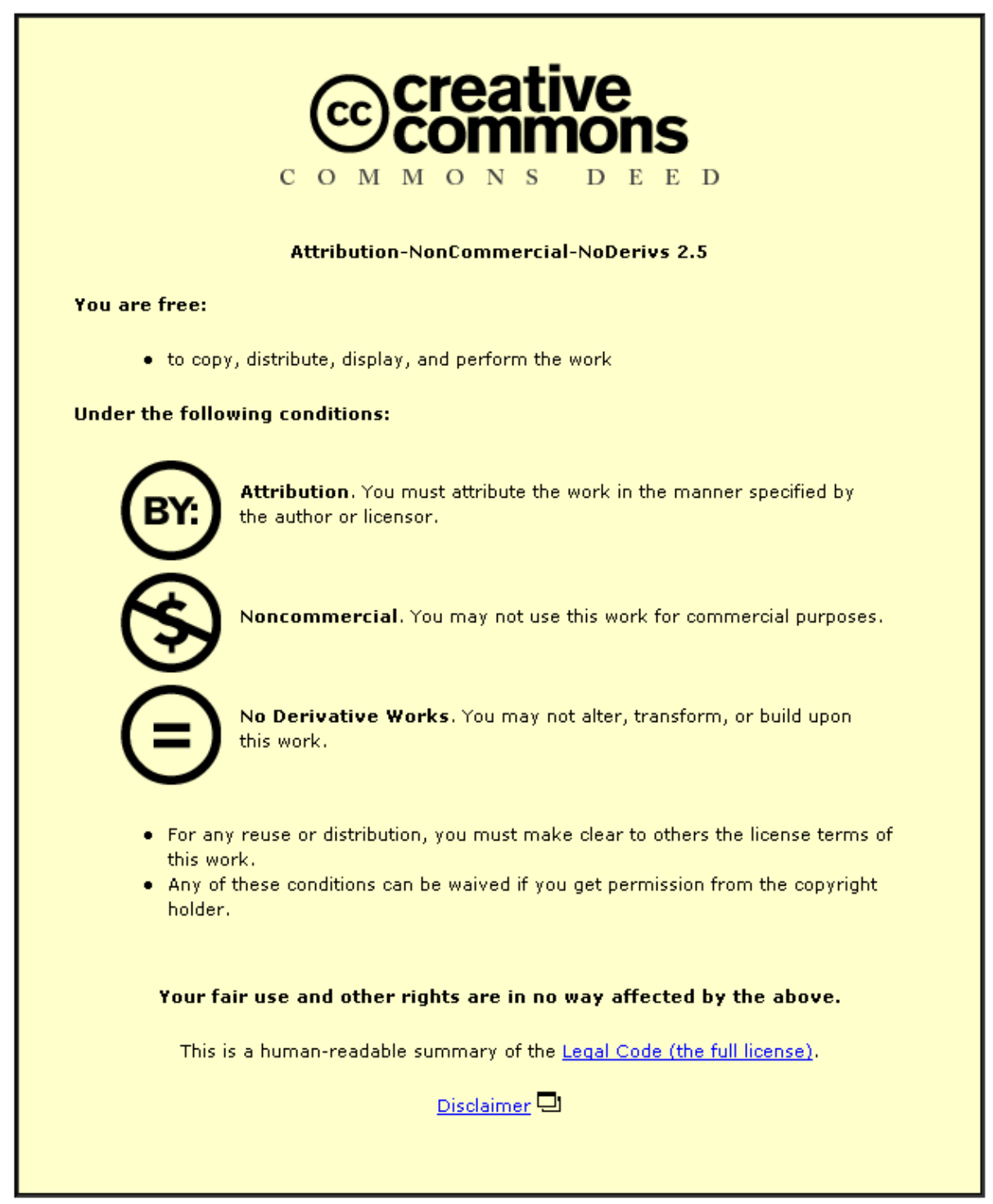

For the full text of this licence, please go to: http://creativecommons.org/licenses/by-nc-nd/2.5/ 


\title{
A single representation to support assembly and process planning in feature-based design machined parts
}

\author{
K Case $^{1}$ and W A Wan Harun ${ }^{2}$ \\ ${ }^{1}$ Department of Manufacturing Engineering, Loughborough University, Leicestershire, UK \\ ${ }^{2}$ SIRIM, Shah Alam, Malaysia
}

\begin{abstract}
The need for a product model that can support the modelling requirements of a broad range of applications leads to the application of a feature-based model within a computer aided design environment. An important requirement in feature-based design for manufacture is that a single feature representation should be capable of concurrently supporting a number of different applications. Assembly and process planning are seen as two crucial manufacturing applications and a formal structure for their representation in a feature-based design system is presented. This research addresses two basic questions relating to the lack of a unified definition for features and the establishment of a feature-based representation for assembly. Thus the concept of designing with features is extended by incorporating assembly and process planning information with the geometrical and topological details of component parts. A prototype system has been implemented using an object-oriented programming technique which provides a convenient method for adding functionality to the geometric reasoning process of features and the complex relationships between the parts that make up the assembly. The feature-based model is embedded in the ACIS object-oriented solid modeller kernel.
\end{abstract}

Keywords: feature-based design, product model, assembly, process planning

\section{INTRODUCTION}

The requirement for shorter product life cycles, increased pressure for shorter time to market and demand for high-quality products makes it imperative for manufacturing industry to focus on new product development strategies in design and manufacturing processes. As a consequence, issues such as concurrent engineering and design for manufacturability and assembly (DFMA) have received increasing attention by manufacturing industries. These concepts attempt to address the issue of product development productivity by helping the designer to make early decisions that minimize costs over the life of the product. A critical aspect of implementing these concepts is the integration of design and manufacturing processes to provide computerized decision support tools on the basis of a product model.

Product modelling refers to the activities related to representing and utilizing information related to products, their design and manufacturing processes and their production management [1]. The ultimate goal of product modelling is to be able to represent all this information in a way that makes it possible to capture and access the relevant information throughout the entire range of design, planning and manufacturing activities. The ideal product model should automatically generate the design, functions, service life, manufacturing methods and all data needed for the processing of customer orders [2]. Various modelling approaches have been proposed [3], but, as much of the information needed in the design and manufacturing process deals with the geometric shape of the product, the geometric model forms the most important component in the product model representation. 


\section{FEATURE MODELLING}

In order to manipulate data in the geometric model for design and manufacturing activities, various geometric reasoning techniques have been developed. Geometric reasoning involves the application of computer techniques to spatial problems so that deductions can be made from geometry [4]. To facilitate geometric reasoning, part geometry must be represented by higher-level entities that relate directly to design functionalities or manufacturing characteristics. The concept of features has been proposed to serve the geometric reasoning needs of CAD systems. Instead of using a model consisting purely of geometric primitives, the designer uses a set of features such as holes, pockets and slots. The growing use of features in the CAD/CAM area is because features offer many advantages over conventional CAD systems, as identified by many authors (e.g. references [1] and [5-9]). Features provide a natural vocabulary to capture the design intent, facilitate the management of parameter relationships and dependences in a model and provide a basis for modelling various manufacturing planning information and can have benefits in standardization.

Features provide an alternative component representation that is suitable for a wide range of activities, thus bridging the gap between design and manufacturing. Because of this potential, features have been used in many CAD/CAM applications [10] such as design [11], process planning [12,13], casting [14], injection moulding [15], design for assembly [16], assembly planning [17], inspection planning [18] and manufacturing cost analysis [19].

A significant aspect of the development of these applications is that a system is typically only capable of supporting a single specific application domain. This limitation is mainly due to the way the features are defined and the data represented. The ability of a feature-based representation to support more than one application is important in a concurrent engineering environment and to fulfil the requirement of a product model that can support the product development process.

Many existing CAD/CAM packages can be classified as geometric modellers with data structures designed to manipulate individual parts. However, in most engineering design the product of interest is a composition of parts formed into an assembly and there is a need for a system that allows a designer to create individual parts, assemble them and then perform the necessary analysis of the assembly. Modelling and representing assemblies, generating assembly sequences and analysing assembly are all relevant issues for geometric modelling and CAD/ CAM technology.
Very early work [20] had established experimentally that a feature-based interface performed better than a purely geometric one for the detailed design aspects of machined components, and this is reflected by the widespread existence of feature 'front-ends' to modern CAD systems. This performance increase was measured in terms of speed, accuracy and completeness of the modelling, but parallel subjective studies established that the industrially based designers forming the experimental group could also see the potential value of the process planning information inherent in the manufacturing features. Subsequent research by the authors $[\mathbf{1 3}, 21]$ established a feature-based representation for machined piece parts based on a previously proposed taxonomy [22]. Central to this representation was the concept of a feature being a volume of material to be removed that is enclosed by a set of real and imaginary (closure) faces. The real faces represent an end condition that must be achieved by machining from the stock material, and the imaginary faces represent external access directions (EADs) or directions for tool access. Taken together, a set of EADs having the same direction vector form a potential access direction (PAD) used in set-up determination. In this way a set of features on a single part could be associated for simultaneous or consecutive machining. This representation was shown to be effective in process capability modelling used for processing equipment selection [23] and formed the basis for grouping of a large number of components from the database of an industrial collaborator for cellular manufacturing [24].

These activities clearly demonstrated through the building of prototype systems and their use in experimental and case study work that it was possible to support a single activity (process planning in this case) by a feature-based representation that had been specifically fashioned to meet the needs of the application. At the same time other researchers (detailed above) were establishing similar relationships between features and other separate activities (such as assembly). The major objective of the research reported here was to investigate the use of a single representation for more than one activity. The previously developed feature representation formed the basis for an enhanced representation that accommodates relationships between features on different parts and could thus be used as a method for holding assembly information.

\section{ASSEMBLY MODELLING}

Assembly modelling deals with the interrelations among assembled parts rather than the detailed shapes of each part. Functional understanding of assembly modelling is a key step towards a real CAD 
environment that can support early design [9]. The capability to represent products composed of assemblies is needed to support further integration of manufacturing systems at a more general level as well as to serve the information needs of the applications at the level of the part [25]. An assembly model provides data for generating assembly sequences and for assembly analysis.

A mechanical assembly can be represented by the description of its individual parts and their relationships in the assembly. Most of the interaction between parts occurs at mating surfaces. The modelling representation of these relationships and mating conditions are the distinguishing characteristics between modelling single parts and assemblies. Thus an assembly modeller can be considered as an extension of a geometric modeller where the data structure is enhanced to allow representation and manipulation of part relationships and mating conditions.

Individual parts are first created with the shape information (geometry and topology) and are then analysed and assembled. An ideal system allows the link to be established between the geometric and assembly models such that designers need only modify individual parts for design modification by using the geometric modeller and the assembly model is updated automatically [26].

Owing to the importance of assembly modelling, the activity has been the subject of much research work related to geometric modelling [27]. Various assembly representation schemes are possible, but the use of features instead of piece parts as the lowest denomination of a product is increasingly popular. This is because feature-based design has been found to facilitate assembly modelling applications by providing natural semantics for describing part interactions in a CAD system.

Features in this research are defined as machined volumes which are represented in a hierarchical taxonomy (for details see reference [22]). The taxonomy includes several types and profiles of features which cover a general range of machined parts. A hierarchical assembly structure has also been defined in which features form basic entities in the assembly. Each feature includes information needed to establish assembly relationships among features in the form of mating relationships. An analysis of typical assemblies has clearly shown that assembly interfaces occur at the face level of the mating features and between features themselves. Three mating relationships between pairs of features have been defined and are represented in the form of expressions that can be used for evaluations. Data on the assembly relationships are combined with knowledge on process planning from which an integrated data structure is derived. This forms the basis of defining classes for each level in the assembly hierarchy.

\section{ASSEMBLY STRUCTURE AND MATING RELATIONSHIPS}

An assembly database stores the geometric models of individual parts, the spatial positions and orientations of the parts in the assembly and the assembly relationships between parts. Zeid [26] provides a comprehensive description of such information models and the uses to which they are put, but many representation schemes have been developed, the main differences being the way in which the locations and orientations of the parts and their hierarchical relationships are represented. Most use a hierarchical structure, and Wang and Ozsoy [28] are typical in using an assembly graph for hierarchical representation of the assembly, its subassemblies and components where the mating conditions of 'against', 'fit' and 'parallel' are stored as a set of mating links. Spatial information is typically represented either explicitly or implicitly. Explicit representation requires that all subassemblies and parts have to be provided with a suitable transformation matrix, whereas implicit representation allows the transformation to be determined from the geometry and mating conditions. Implicit representation has the very significant benefit of allowing the designer to manipulate the model via the mating conditions and thus separates control of geometric aspects of the model from configuration of the assembly.

In the present work an analysis of a number of assemblies was undertaken to determine a suitable set of mating conditions that could be used to build an implicit representation within a hierarchical structure. Example assemblies are shown in Fig. 1. These and other examples have been analysed to determine the assembly interactions so that a set of mating conditions can be defined and methods devised for their representation in a structure. The first stage of the analysis establishes relationships between the components that go to make up the assembly and these are expressed in a component relation graph. This is a frequently used way of describing assemblies and Fig. 2 shows the component relation graph for the lathe tool post. Physical interactions between the components are identified and labelled (Fig. 2a) and from this information a graph of the relationships can be formed (Fig. 2b). At this time only the existence of a relationship has been established and the type of such relationships remains to be determined. Component relation graphs are useful in assembly planning as they capture information that is useful in determining the creation of subassemblies. For example, it is clear from Fig. $2 b$ that the set screws could be assembled to the tool post to form a subassembly. Similarly, components that might be considered as candidates for being the base part to which other components might be assembled could be identified as those having the most relationships (the top 
slide in this case). Particular rules for assembly are not, however, based on these kinds of considerations alone. In particular it is also necessary to have knowledge of the type of relationship and the geometric conditions.

In the present work three basic mating relationships were determined from the analysis of example assemblies to confirm the usefulness of those defined in the literature. Against and Fits are relationships originally defined by Lee and Gossard [29] and later used by Wang and Ozsoy [28], and Aligns is a useful composite relationship as described below. Against is a relationship between faces that in geometric terms ensures that the normal vectors to the two faces are in opposing directions. This is most commonly found where both faces are planar (e.g. between the bottom of the tool post and the top of the top slide), but other face types (e.g. cylindrical) are within the representation scheme. Fits is a mating relationship that occurs when two features are required to fit together with clearance or interference (e.g. the tee bolt into the tool post hole). A Fits relationship typically generates a colinearity condition on the centre-lines of the two features and can be subclassified according to the method or difficulty of assembly (i.e. tight fit, screw fit, clearance fit). The Align relationship exists, for example, where centre-lines of two holes must coincide (e.g. bolt holes on the two halves of the valve subassembly in Fig. 1a) or where two planar faces must lie on the same surface (e.g. the faces of the top slide boss and the boss of the tool post). The significance of these mating relationships is not only in being descriptive of the assembly process but also in forming an essential part of the geometric reasoning required to maintain an implicit representation of the spatial relationships between components of the assembly.

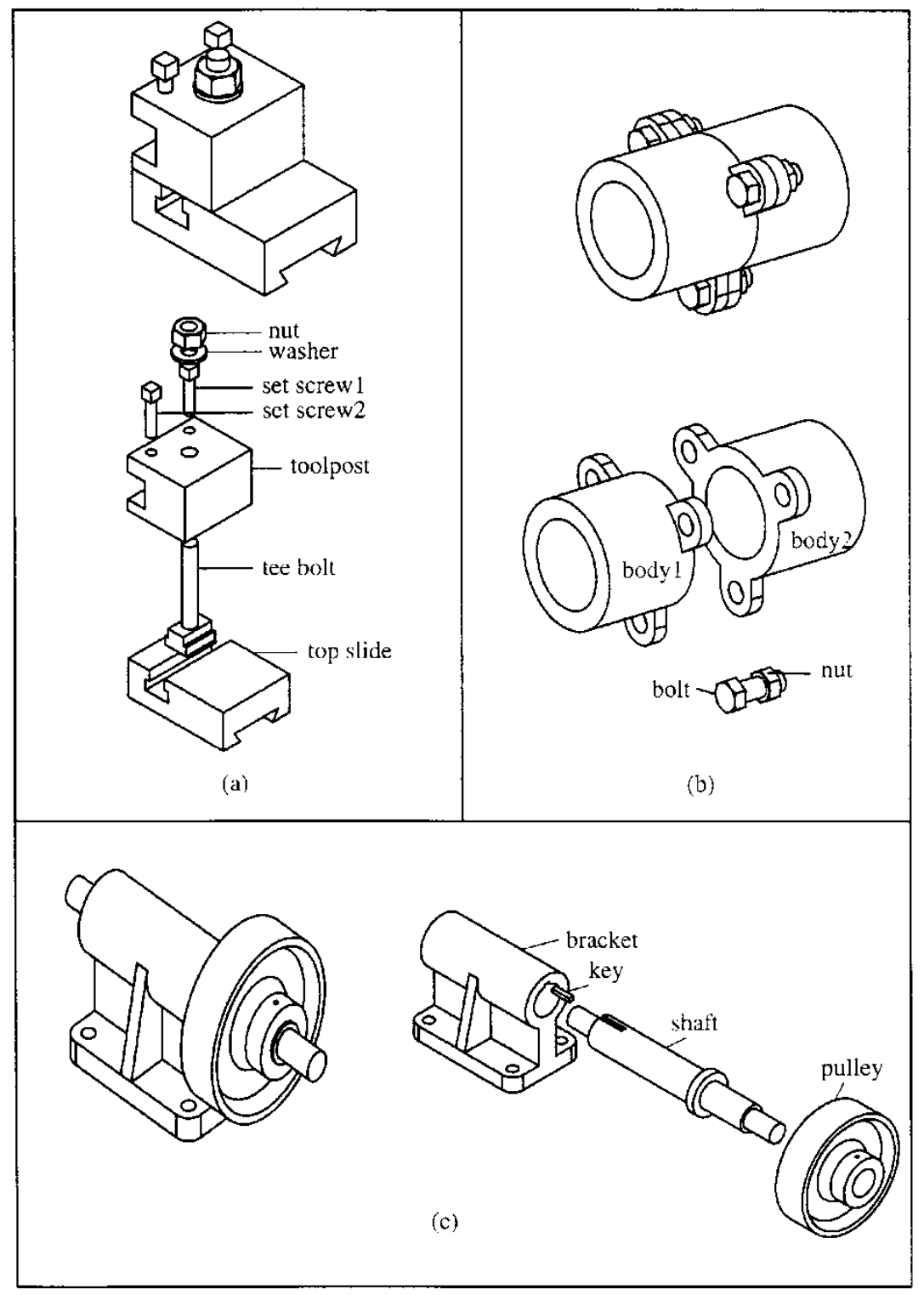

Fig. 1 Examples used in mating conditions analysis: (a) lathe tool post; (b) valve subassembly; (c) bracket and pulley 


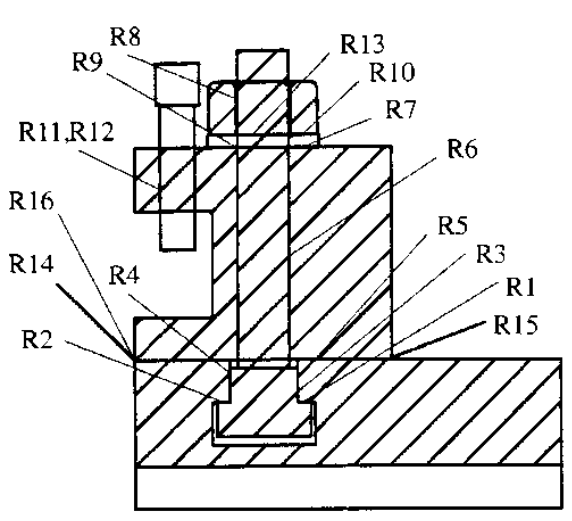

(a) Component Relationships

Key to Relationships

R1: step of tee-bolt (right)

R2: step of tee-bolt (left)

R3: vertical side of tee-bolt (right)

R4: vertical side of tee-bolt (left)

R5: bottom face of tool post

R6: tee-bolt

R7: bottom face of washer

R8: tee-bolt

R9: tee-bolt

R10: top face of washer

R11: shaft of set screw 1

R12: shaft of set screw 2

R13: hole of nut

R14: left side of tool post

R15: front side of tool post

R16: back side of tool post

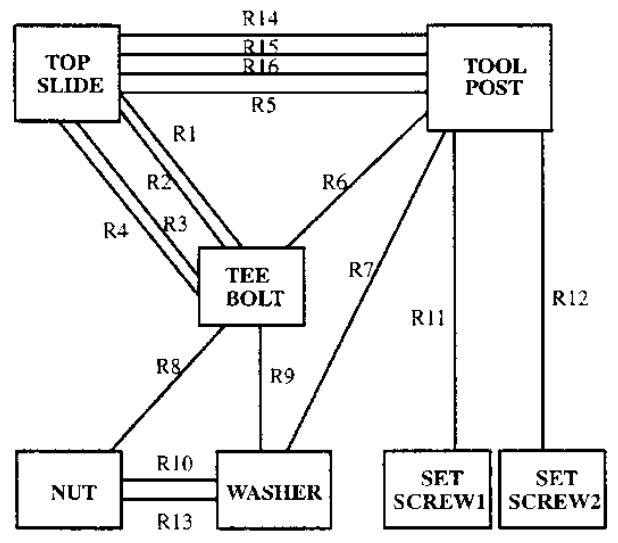

(b) Relationship Graph

through slot of top slide through slot of top guide through slot of top guide through slot of top guide top face of top slide hole of tool post top face of tool post hole of nut

hole of washer bottom face of nut hole of tool post hole of tool post hole of washer left side of top slide front of top slide back of top slide

Fig. 2 Component relation graph for the lathe tool post assembly

\section{FEATURE-BASED ASSEMBLY MODELLING}

The discussion of mating relationships in the previous section clearly demonstrates that the component relation graph (and its manifestation in a data structure) is inadequate for complete representation of the situation. It is features of components that are important to assembly, and not just components themselves. Hence there is a need for the further level of detail provided by the feature relation graph (Fig. 3). In this graph each component is represented by a set of features and the mating relationships are now shown as existing between features. A feature relation table (Table 1) consists of a matrix of possible feature relationships from which it can be determined (for example) that relationship R5 in Fig. 3 must be either Aligns or Against. The addition of geometric conditions determines that it is actually Aligns.

For some conditions the feature -feature relationship might be capable of fully specifying the configuration of components in the assembly, but frequently the more detailed information provided by the face mating graph (Fig. 4) is required. Faces can be considered as subfeatures that play an important role in representing assembly information. Equally importantly, faces are crucial to the manufacturing of the components as the end result of machining and as the carriers of process planning information such as dimensions and tolerances. Features and faces together form the important common ground between the assembly planning and process planning activities and hence the objective of a single representation for multiple applications can be achieved. The face mating graph contains all the topological information relating components, features and faces and can be simply extended to include any level of subassemblies. It has also been extended to the component connectivity graph (e.g. Fig. 5) which is essentially of the same form but with additional process planning relationships (tool access directions, set-up directions, dimensions and tolerances) represented at the face-to- 
face level. Thus the current information structures subsume those from earlier process planning work [21].

\section{ASSEMBLY AND PROCESS PLANNING FEATURES}

Three types of mating condition have previously been identified which associate pairs of machining features. As features presented in this research are machining features that have been used for process planning [13], knowledge on process planning has been associated with each feature. In order to find the relation between the assembly relationships and the process planning information, assemblies at the face level for selected parts in the three example products (Fig. 1) are re-examined, but before doing so a brief description of the aims of process planning will be given.

The overall objective of process planning is to devise a method of manufacture that is optimal with respect to a set of criteria such as economy and quality, although it is recognized that the ideal condition of optimality cannot be attained in practice. Some of the key aspects of process planning are overall process selection (e.g. machining, forming or fabrication), specific machining process selection (e.g. turning, milling or grinding), machine selection (a specific machine from those available), set-up determination, operations sequence planning (order of machining at a particular set-up) and machining parameter selection (feeds, speeds, etc.). A successful representation must be capable of maintaining information relevant to these activities and their interrelationships together with the previously discussed assembly relationships.

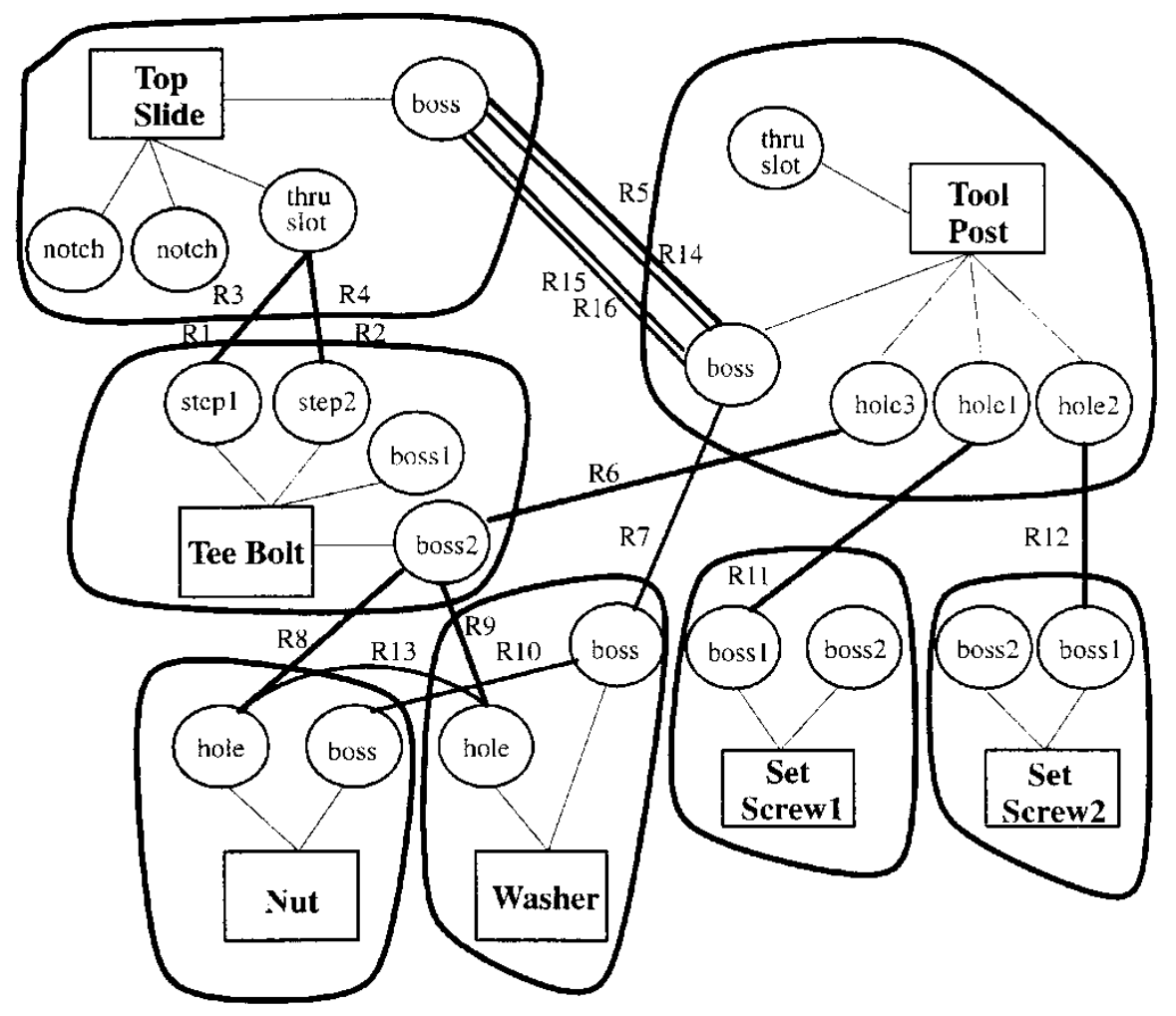

Fig. 3 Feature relation graph for lathe tool post assembly

Table 1 Feature relation table

\begin{tabular}{lllllllll}
\hline & Boss & Hole & Pocket & Thruslot & Non-thruslot & Notch & Step & Surface \\
\hline Boss & A, L & F & F, L & A & A & A & A & A \\
Hole & F & L & L & X & X & X & X & X \\
Pocket & F, L & L & L & X & X & X & X & X \\
Thruslot & A & X & X & L & X & X & A & A \\
Non-thruslot & A & X & X & X & X & X & A & A \\
Notch & A & X & X & X & X & A & X & A \\
Step & A & X & X & X & A & X & A, L & A, L \\
Surface & A & X & X & A & A & A & A & A, L \\
\hline
\end{tabular}

Key: $\mathrm{A}=$ Against; $\mathrm{F}=$ Fits: $\mathrm{L}=$ Aligns; $\mathrm{X}=$ no relationship. 


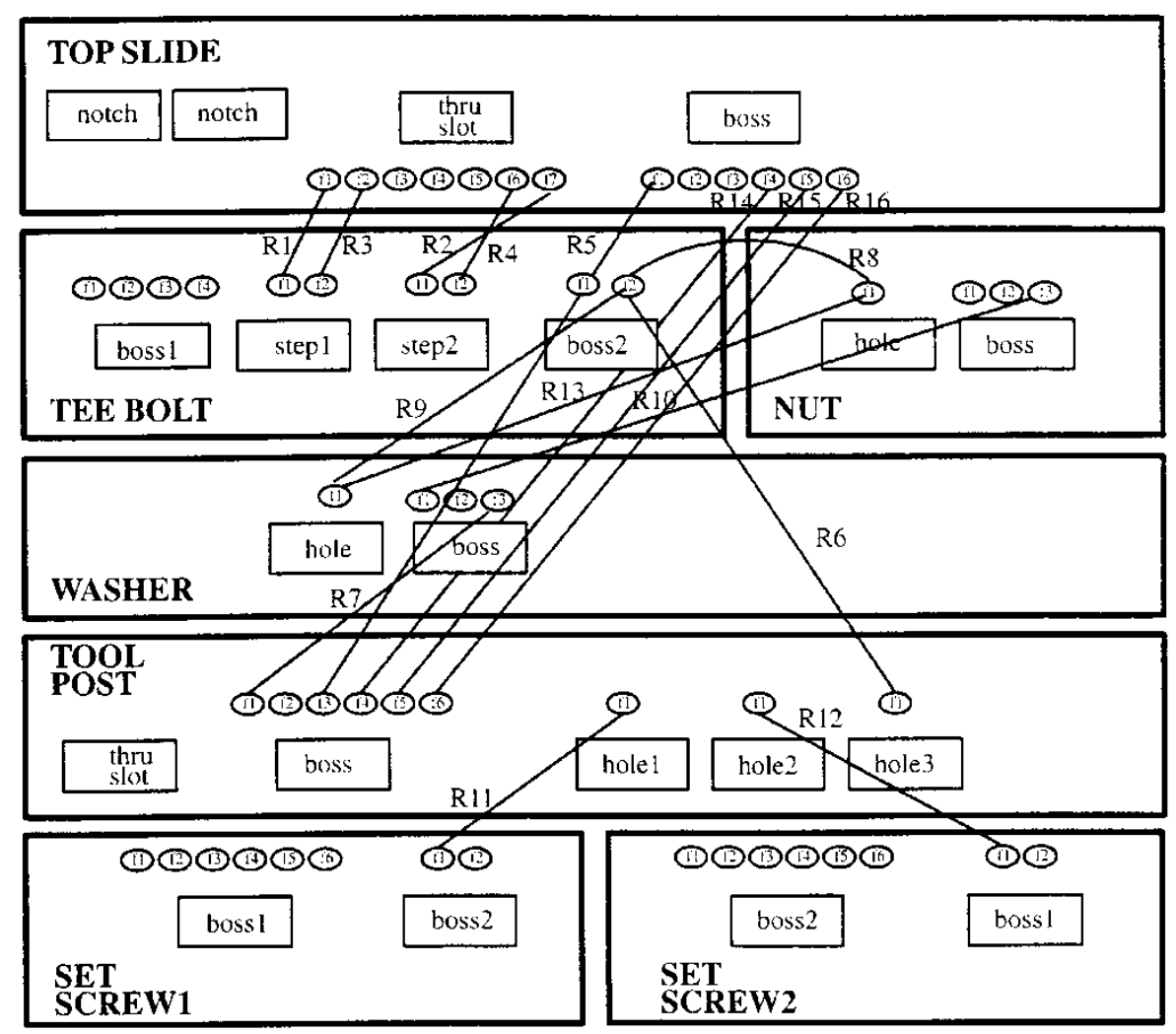

Fig. 4 Face mating graph for lathe tool post assembly

Thus the process planning task requires a considerable amount of information about the parts to be manufactured. In a feature-based process planning system, information is inferred from the feature model data. The process planning information that is required for each feature and relevant to this work is as follows:

1. The dimensional and geometric tolerances are important to ensure that parts will function correctly, be interchangeable and can be manufactured economically. Dimensional tolerances, marked $\mathbf{d}$ in the subsequent figures, are used to communicate ranges of dimensions that are acceptable in meeting functionality. Geometric tolerances such as parallelism (marked // in the figures), circularity (C) and flatness (F) further refine the specification for manufacturing to meet functional requirements.

2. The imaginary faces, represented as $i_{n}$, determine the external access directions (EADs), which are potential tool approach directions in machining, and can be used in set-up determination.

3. The surface finish attribute can be used in determining the suitable manufacturing process.

4. The parent-child relationship determines the machining precedence. It also affects the tool access directions, operation sequencing and set-up strategy. A parent -child relationship exists if one feature can be defined with respect to another feature. The former is called a child while the latter is a parent feature. For example, with a countersunk hole, the hole might be the child of the countersink or vice versa.

These items of information are added to the selected parts in each assembly and its relation with the assembly relationship is examined. These are shown in the component connectivity graphs in Figs 5, 6 and 7. The value of these component connectivity graphs can be seen through the extent to which they fulfil the information requirements of both process and assembly planning. Using Fig. 5 as an example, a geometric modelling system would normally supply the face level information and in some cases might be capable of expressing groups of faces as features (through slot, boss and two steps) and groups of features as components (top slide and tee bolt). However, conventional systems would be unlikely to have the capability of expressing the 'between face' relationships shown as arcs in the figure. An assembly modeller should be capable of describing a set of constraints (mating conditions) that specify a unique spatial and functional configuration for the assembled product. In this example the tee bolt is required to have one degree of translational freedom to meet the functional requirement, with the remaining five degrees of freedom constrained to represent the assembled location. There may be several ways of achieving this with different sets of mating conditions and Fig. 5 illustrates one possibility using Against conditions. The component 
connectivity graph provides information that is useful in analysing certain aspects of the validity of the way in which mating conditions have been expressed. In this case, for example, the use of Against conditions requires that real rather than imaginary faces are involved in the relationship and this can be confirmed by the face attributes held within the feature representation. In addition to being capable of representing an assembled state, a useful assembly modeller will also be able to provide information for determining the feasibility of attaining this state. Much of this information resides in the geometric model and is used to determine interference conditions, but exhaustive searching for assembly directions can be avoided by a modeller that provides potential assembly directions (PADs). These directions are defined by sets of real faces on one component entering the imaginary faces of the mating component. In this example the top slide has three imaginary faces (the two T-shaped ends and the rectangular top), each of which is a potential assembly direction. Figure 5 actually shows face $i_{2}$ of the through slot as not being suitable for assembly and this is intended to represent some post-processed situation where geometric tests for interference have precluded this as a PAD.

Process planning information comes in part from the needs of component manufacture and therefore, as previously described, each imaginary face represents a potential way of gaining access for machining. These are identified as external access directions (EADs) in Fig. 5 and it is clear from the figure that machining access directions (EADs) are closely related to assembly directions (PADs). Sets of EADs with a common normal

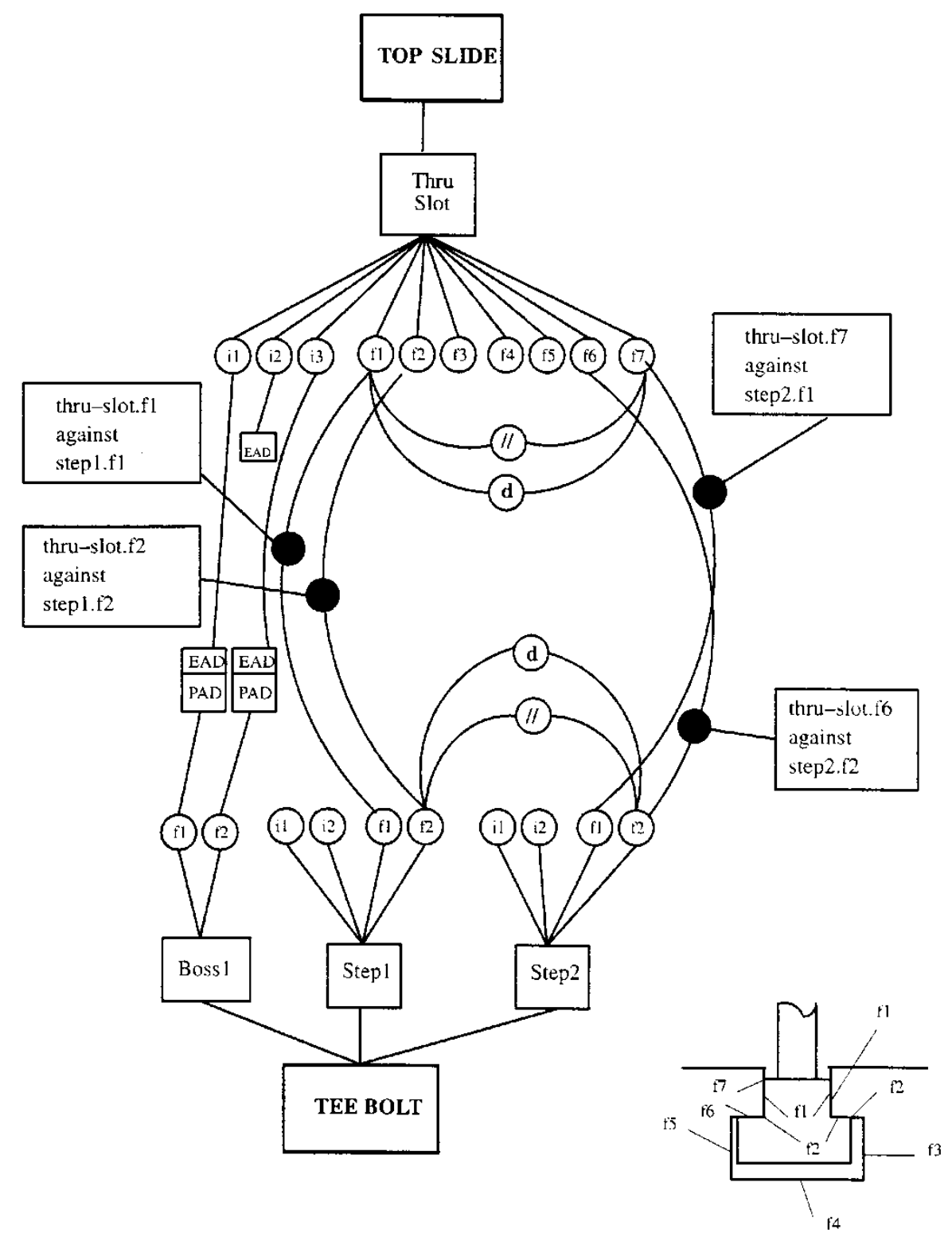

Fig. 5 Component connectivity graph for lathe tool post assembly 


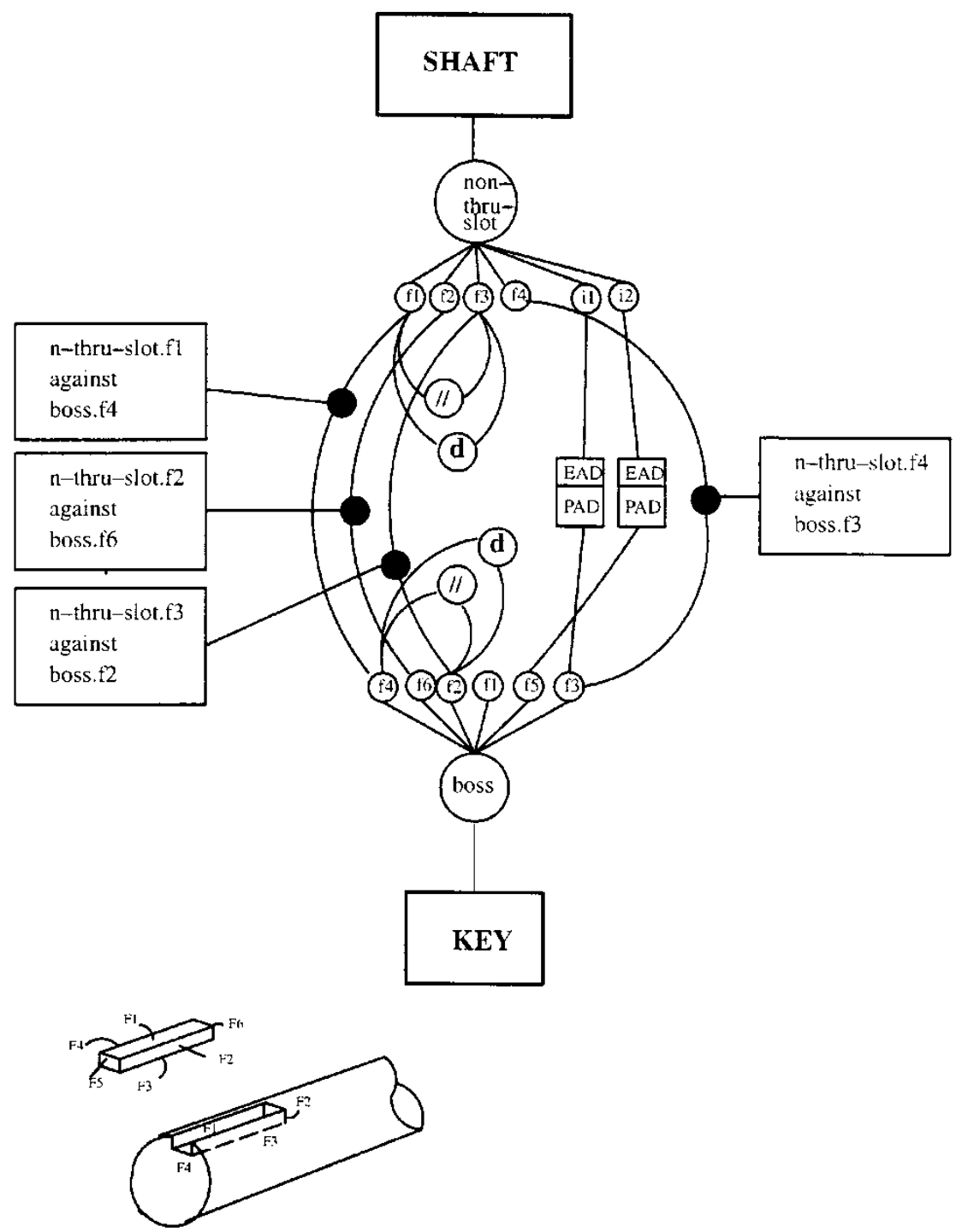

Fig. 6 Component connectivity graph for key and keyway

vector represent potential machining set-ups (i.e. groups of features that can be machined from the same direction). Assembly imposes some process planning considerations through the dimensions and tolerances required to meet the needs of the mating conditions. Thus, for example, the Against mating conditions require an adequate set of geometric conditions on the components and these are expressed as dimensional and geometric tolerances between faces of different features and faces of a single feature. Once again there may be several alternative sets of tolerances and, in searching for these, the component connectivity graph shows that each face involved in a mating condition will potentially carry a dimensional and geometric tolerance. Figure 5 shows one such set that fulfils the mating requirements.

The assembly of a key to a shaft is shown in Fig. 6 and illustrates similar points. Each of the imaginary faces (there are only two) are both external access directions for machining and potential assembly directions. The Against mating conditions are again relationships between real faces of different components, and the dimensional tolerances and parallel conditions are an illustrative set of component conditions that must be process planned in order to achieve the assembly.

Figure 7 shows the interactions between four parts in the valve subassembly and illustrates use of the Fits and Align mating conditions. Of the four imaginary faces (two on the nut and two on the body), two are shown as external access directions for machining and potential assembly directions, while it is implied that the remaining two have been excluded from performing these functions by some geometric analysis. The Align mating condition between the body and the nut is accompanied by circularity geometric tolerances on both holes and the Against condition between the body and the bolt is accompanied by a flatness geometric tolerance on one face of the body. 
From the above analysis, the following observations, summarized in Fig. 8, are inferred:

1. The external access direction (EAD) of each feature can be viewed both as a potential machining direction and a potential direction in which another feature can be assembled to it. The latter direction is referred to as a potential assembly direction (PAD) and occurs between an imaginary face of one feature and a real face of a mating feature.

2. Each mating face has its own process planning data attached to it. These data either relate to the feature itself, such as the cylindricity of a hole, or they represent the relationship between two features such as the parallelism of the sides of the features.

3. Some of the process planning data are relevant to the assembly modelling. For example, the dimen- sional tolerance will determine the type of fit between two features. For example, the parallelism of the faces is important if two faces are to have sliding contact, as shown by the example of the assembly of steps to the through slot in the lathe tool post assembly. Other information such as parent - child relationships is not relevant to the assembly modelling and is only used to determine intermediate configurations of the component during machining.

4. The component connectivity graphs show a clear relationship between process planning and assembly information. For example, the functional assembly requirement of the tee bolt to mate with the through slot of the top slide (Fig. 5) generates the assembly information describing Against conditions between the faces of the components. Process planning infor-

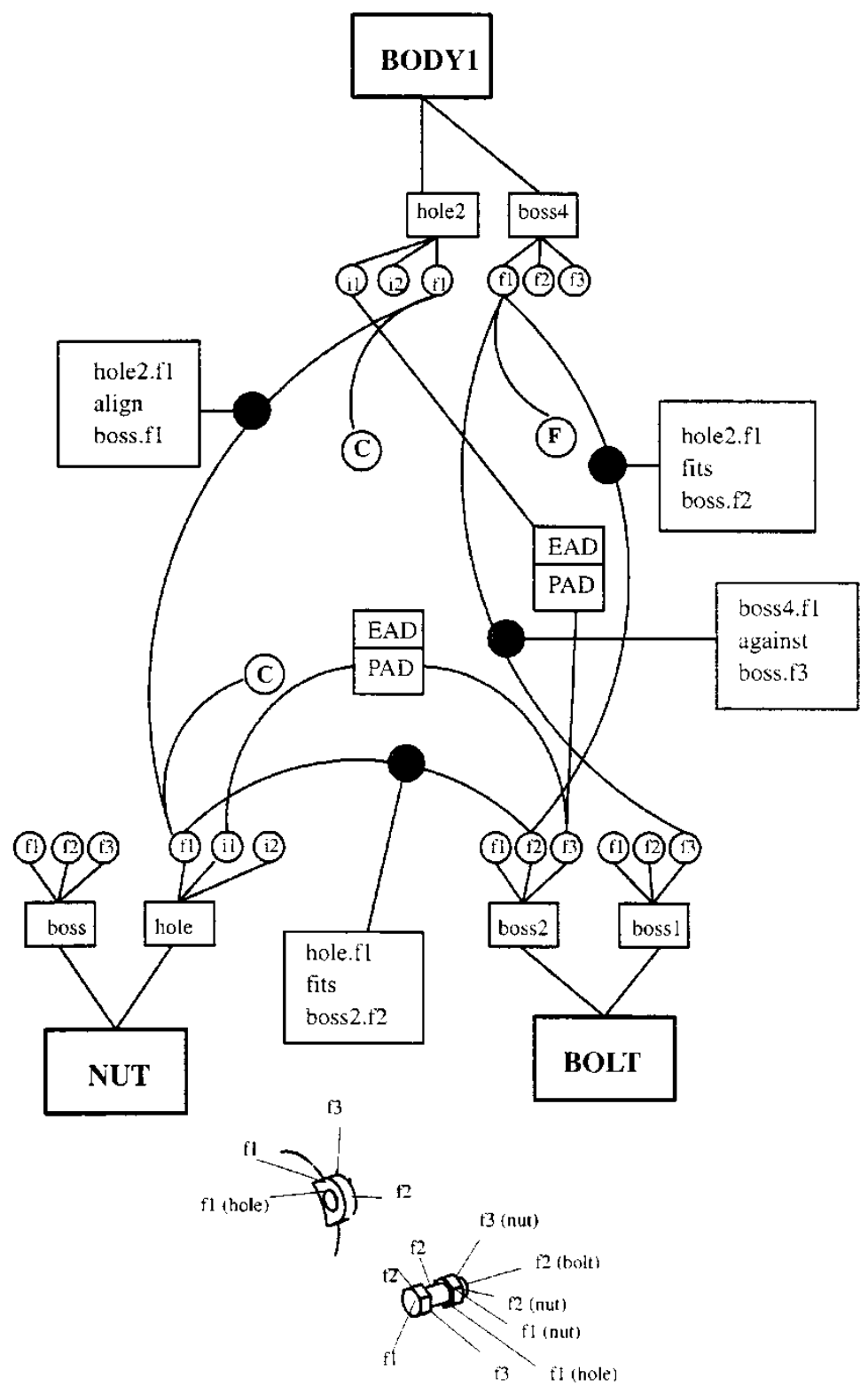

Fig. 7 Component connectivity graph for valve subassembly 


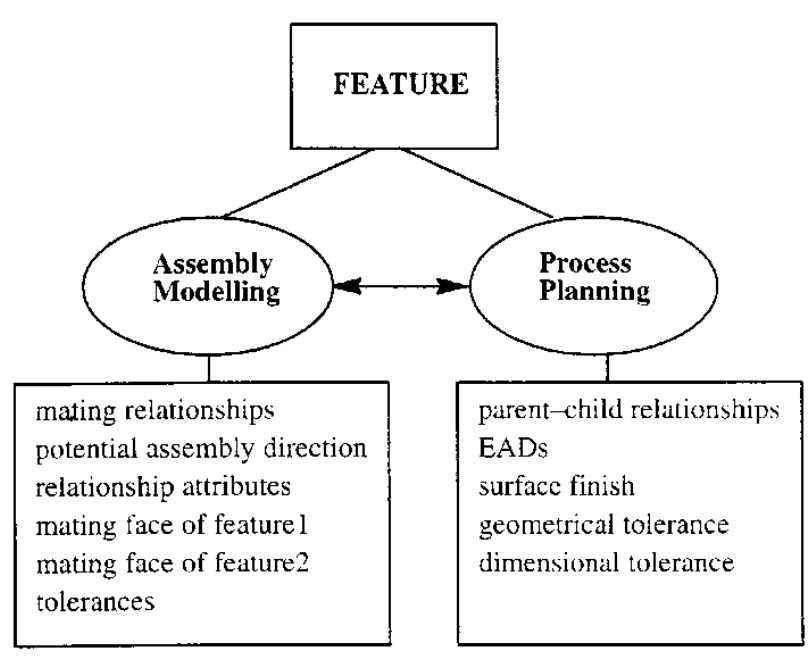

Fig. 8 Relation between assembly and process planning knowledge in a feature

mation in the form of dimensional and geometrical tolerances on the faces of individual features of each component are then required to ensure this assembly functionality.

To realize the benefits of a combined assembly and process planning representation it is necessary to implement it as a data structure in a feature-based model, and this is discussed in the next section.

\section{IMPLEMENTATION}

Suitable data structuring provides an important link between the assembly database and the database of its assembled parts so that when any part (a feature, a component or a subassembly) is modified the corresponding instance in the assembly is updated automatically. Linked lists are one of the basic elements of $\mathrm{C}++$ programming and offer several advantages over other structures such as arrays. Lists do not have predefined size and they can be formed, reorganized or destroyed dynamically, object by object, using defined pointers. This is useful in the feature-based modelling situation where features are interactively added, moved or deleted from the components or subassemblies. Lists are also claimed to be fast and fit the object-oriented way of thinking.

An assembly can be considered as a list of subassemblies. Each subassembly is a list of components, a component is a list of features and a feature is a list of faces in the geometric model. This implies the use of a linked list to represent each level of the assembly. The general form of the assembly data structure is shown in Fig. 9, and that for a component in Fig. 10, both as linked lists using forward ring pointers. The assembly level (Fig. 9) contains the following information:

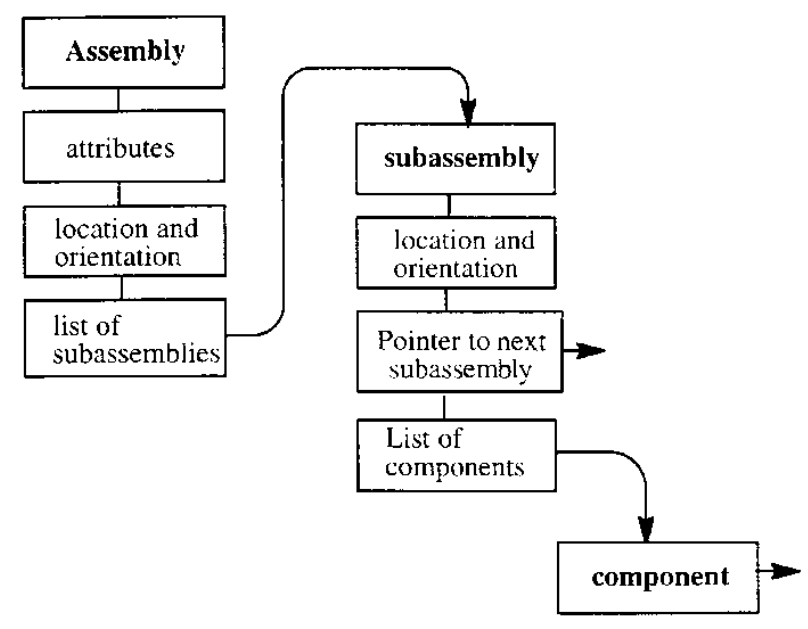

Fig. 9 Data structure for assembly and subassembly levels

(a) name of assembly (e.g. lathe tool post),

(b) product attributes (e.g. mass, overall tolerance, etc.),

(c) location and orientation of the assembly with respect to a world coordinate system,

(d) pointer to a list of subassemblies.

A subassembly has a similar information structure, including:

(a) name of subassembly (e.g. top slide),

(b) location and orientation of the subassembly with respect to a world coordinate system,

(c) pointer to the next subassembly that is also part of the assembly,

(d) pointer to a list of components that constitutes the subassembly.

Referring to Fig. 10, the data structure for the component consists of the following information:

(a) component name (e.g. bolt),

(b) component attributes (e.g. mass, material, overall dimensions, etc.),

(c) location and orientation with respect to a world coordinate system,

(d) pointer to next component in the assembly,

(e) pointer to a list of features that constitutes the component,

(f) pointer to tolerance relationships,

(g) pointer to parent -child relationships.

For an individual feature, the parameters refer to the dimensions and the number of external access directions. The face list refers to the list of mating faces which is accessible from the geometric model created in ACIS.

The data structure for the mating relationships contains the following information:

(a) pointer to feature 1 ,

(b) pointer to feature 2, 
(c) type of mating relationship (mating feature),

(d) relationship attributes (e.g. screw or sliding fit),

(e) pointer to the mating face of feature 1 ,

(f) pointer to the mating face of feature 2 .

In the above structure, a mating between a pair of features is represented by a pointer to the feature and a pointer to the mating face. In the event of one feature being removed from the assembly or a new feature being added to mate with an existing feature, the pointer will be reset to point to the new object.

The assembly representations described above have been implemented by means of the ACIS solid modelling kernel using a $\mathrm{C}++$ object-oriented approach. The details of the implementation are beyond the scope of this paper, but classes have been created for assemblies, features, feature types, profiles, feature relationships, etc., together with appropriate methods for their creation, manipulation and use within the ACIS environment.

\section{CONCLUSIONS}

The research presented here reinforces the idea that features can be used in multiple applications and that the object-oriented approach assists in moving towards a unified definition for features. Features which have previously been used for process planning have been used in an enhanced form to represent assemblies. The use of features for assembly modelling provides a natural representation, since in assembly operations it is the feature that dictates the way in which parts are assembled. The approach adopted provides a design tool for designers by allowing them to create a mechanical

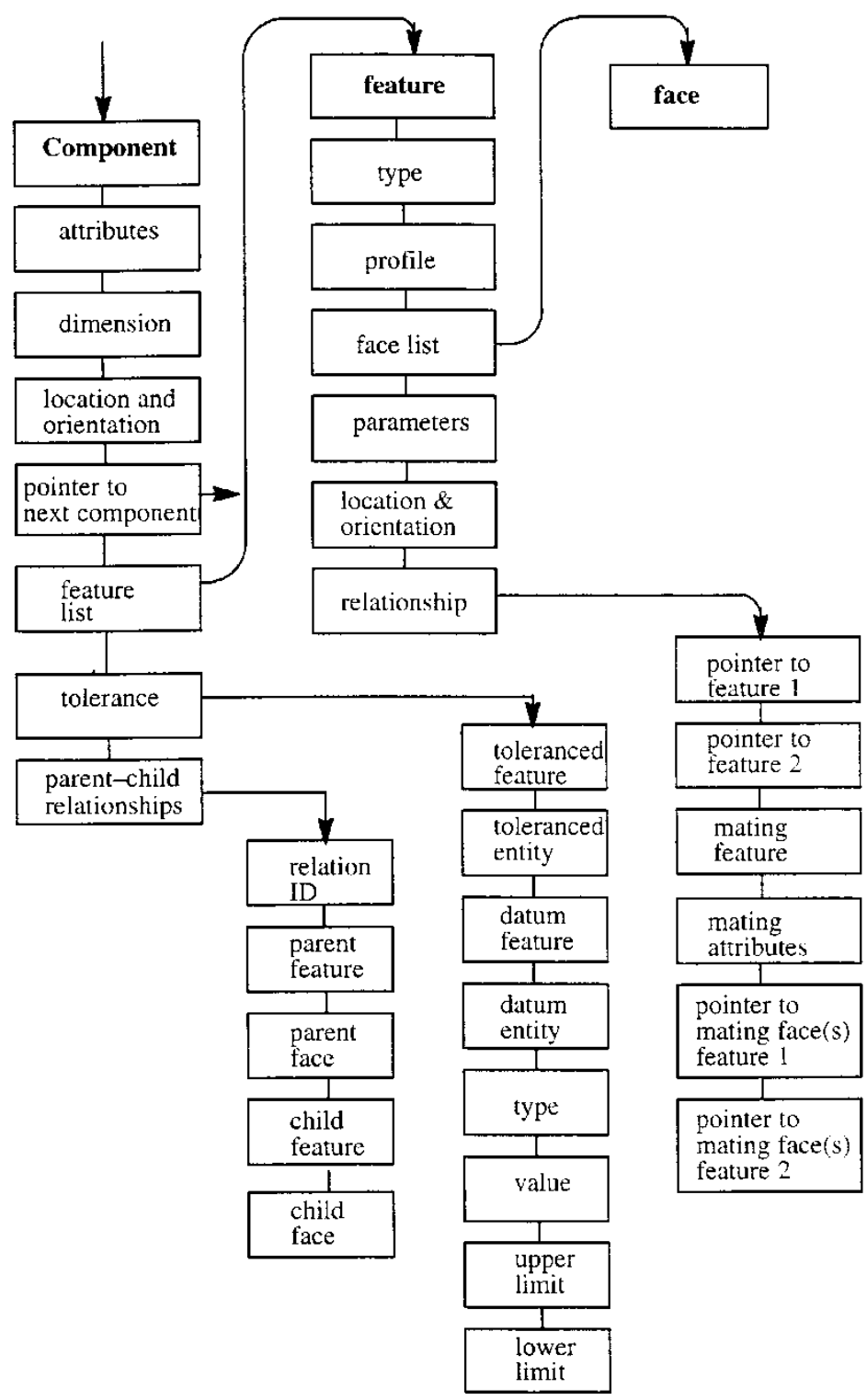

Fig. 10 Data structure for component and feature levels 
assembly in terms of features, which is applicable for subsequent manufacturing planning activities. The feature representation methodology implemented is suitable for the concurrent representation of knowledge on process planning and assembly modelling. Clearly, this does not conclusively establish that all aspects of design and manufacturing can be encapsulated in a single representation, but it goes some way to confirm the feasibility of the idea.

\section{REFERENCES}

1 Mantyla, M. Directions for research in product modelling. In Computer Applications in Production and Engineering (Eds F. Kimura and A. Rostaldas), IFIP, 1989 (Elsevier Science Publishers).

2 Rembold, U., Nnaji, B.O. and Storr, A. Computer Inte grated Manufacturing and Engineering, 1993 (AddisonWesley).

3 Krause, F.L., Kimura, F., Kjellberg, T. and Lu, S. C. Y. Product modelling. Ann. CIRP, 1993, 42(2), 695-706.

4 Bonney, M.C., Taylor, N. K. and Case, K. Using computer-aided design and expert systems for human workplace design. In Geometric Reasoning (Ed. J. Woodwark), 1989, pp. 269-282 (Clarendon Press, Oxford).

5 Clark, A.L. and South, N.E. Feature-based design of mechanical parts. In Proceedings of AUTOFACT '87, Michigan, 1987 (SME).

6 Shah, J.J., Sreevalsan, P., Rogers, M., Billo, R. and Mathew, A. Current status of features technology. Revised Report, Computer-Aided Manufacturing-International, Inc., Texas, November 1988.

7 van Emmerik, M.J.G.M. and Jansen, F.W. User interface for feature modelling. In Computer Applications in Production and Engineering (Eds F. Kimura and A. Rostaldas), IFIP, 1989, pp. 625-632 (Elsevier Science Publishers).

8 Chen, Y.M., Miller, R.A. and Vemuri, K.R. A framework for feature-based part modelling. Trans. ASME, Comput ers in Engng, 1991, 1, 357-365.

9 Gui, J.K. and Mantyla, M. Functional understanding of assembly modelling. Computer-Aided Des., 1994, 26, 435451.

10 Pratt, M.J. Application of feature recognition in the product life-cycle. Int. J. Computer Integrated Mfg, 1993, 6(1 and 2), 13-19.

11 Case, K. and Gao, J. Feature technology - an overview. Int. J. Computer Integrated Mfg, 1993, 6(1 and 2), 2-12.

12 Krause, F.L., Ulbrich, A. and Vosgerau, F.H. Featurebased approach for the integration of design and process planning systems. In Product Modelling for ComputerAided Design and Manufacturing (Eds Turner, et al.), IFIP, 1991 (Elsevier Science Publishers).

13 Gindy, N.N.Z.. Huang, X. and Ratchev, T.M. Feature- based component model for computer-aided process planning systems. Int. J. Computer Integrated Mfg, 1993, 6(1 and 2), 20-26.

14 Corbett, J. and Woodward, J.P.J. A CAD-integrated knowledge-based system for the design of die cast components. Ann. CIRP, 1991, 40(1), 103-105.

15 Al-Ashaab, A.H.S. and Young, R.I.M. Design for injection moulding in a manufacturing model environment. J. Des. and $\mathrm{Mfg}$, 1995, (5), 45-54.

16 DeFazio, T.L., et al. A prototype of feature-based design for assembly. In Advances in Design Automation, 1990, Vol. 1, DE-Vol 23-l, pp. 9-16 (American Society of Mechanical Engineers, New York).

17 Wang, H.P. and Li, J. K. Computer-Aided Process Planning, 1991 (Elsevier, Netherlands).

18 ElMaraghy, H.A. and ElMaraghy, W.H. Computer-aided inspection planning (CAIP). In Advances in Feature-Based Manufacturing (Eds J. J. Shah, M. Mantyla and D. S. Nau) 1994, pp. 363-396 (Elsevier).

19 Nieminen, J. and Tuomi, J. Design with features for manufacturing cost analysis. In Product Modelling for Computer-Aided Design and Manufacturing (Eds Turner, et al.), IFIP, 1991, pp. 317-330 (Elsevier Science Publishers).

20 Case, K. and Acar, B.S. Learning studies in the use of computer aided design systems for discrete-parts manufacture. Behaviour and Inf. Technol., 1989, 8(5), 353-368.

21 Case, K. Using a design by features CAD system for process capability modelling. Computer Integrated $\mathrm{Mfg}$ Systems, 1994, 7(1), 39-49.

22 Gindy, N.N.Z. A hierarchical structure for form features. Int. J. Prod. Res., 1989, 27, 2089-2103.

23 Gindy, N.N.Z., Case, K., Ratchev, T.M. and Gao, J. Process capability models for design and selection of processing equipment. In Proceedings of Design and Integrated Production (EPSRC) Research Conference, University of Warwick, 7-9 September 1994, pp. 7-12.

24 Gindy, N.N.Z., Ratchev, T.M. and Case, K. Component grouping for GT applications - a fuzzy clustering approach with validity measure. Int. J. Prod. Res., 1995, 33(9), 2493-2509.

25 Usher, J.M. An object-oriented approach to product modelling for manufacturing systems. Computers and Ind. Engng, 1993, 25(1-4), 557-560.

26 Zeid, I. CAD /CAM Theory and Practice, 1991 (McGrawHill, New York).

27 Libardi, E.C., Dixon, J.R. and Simmons, M.K. Computer environment for the design of mechanical assemblies: a research review. Engng With Computers, 1988, (3), 121136.

28 Wang, N. and Ozsoy, T.M. Automatic generation of tolerance chains from mating relations represented in assembly models. Trans. ASME, Adv. in Des. Automn, 1990, 1, 227-233.

29 Lee, K. and Gossard, D.C. A hierarchical structure for representing assemblies: Part 1. Computer Aided Des., 1985, 17(1), 15-19. 